\title{
APPROXIMATION BY POLYGONS AND POLYHEDRA
}

\section{LÁSZL Ó FEJES TÓTH}

In order to investigate as to what order of magnitude a plane (or a skew) curve can be approximated by $n$-sided polygons, we have to start from a definition of the deviation. One of the usual definitions is as follows: ${ }^{1}$ the deviation $\eta(H, K)$ of two curves $H$ and $K$ is the smallest number $\eta$ for which $H$ is contained in the neighbourhood of $K$ with radius $\eta$ and conversely $K$ is contained in the neighbourhood of $H$ with radius $\eta$. By a neighbourhood with radius $\eta$ of a plane (skew) curve we understand the set of the points of all the circles (spheres) with radius $\eta$ whose centres are the points of the curve.

This notion of deviation satisfies the usual requirements; for example, the triangle inequality is satisfied, that is, if $H, K$ and $L$ are three curves, then $\eta(H, K)+\eta(K, L) \geqq \eta(H, L)$.

Corresponding to a rectifiable Jordan-curve $J$, let us construct the polygon $\bar{P}_{n}$ whose deviation from $J$ is least for all inscribed $n$-sided polygons, $P_{n}: \eta\left(\bar{P}_{n}, J\right) \leqq \eta\left(P_{n}, J\right)$. If we impose further restrictions upon $J$, the sequence $\eta\left(\bar{P}_{1}, J\right), \eta\left(\bar{P}_{2}, J\right), \cdots$ will tend towards zero with order $1 / n^{2}$, that is, the limit value

$$
1 / a=\lim \inf n^{2} \eta\left(P_{n}, J\right)=\lim n^{2} \eta\left(\bar{P}_{n}, J\right)
$$

exists. We shall call $a$ the approximability of $J$ by inscribed ${ }^{2}$ polygons. $a$ is a constant which depends only on $J$. The approximability of a circle of radius $r$ is, for example, $a=2 / \pi^{2} r$.

Interesting problems arise if we consider which curve can be approximated the worst among a certain class of curves, that is, whose approximability is the smallest. We easily obtain,$^{3}$ for example, that among all closed convex curves with continuous curvature of given

Received by the editors May 15, 1947.

${ }^{1}$ See, for example, Bonnesen-Fenchel, Theorie der konvexen Körper, Berlin, 1934.

${ }^{2}$ Similarly in the case of a convex arc we can define the approximability by circumscribed polygons, which equals $a$. The approximability by arbitrary polygons equals $2 a$. In all that follows we restrict ourselves to the case of inscribed polygons and polyhedra.

3 Cf. L. Fejes, Einige Extremaleigenschaften des Kreisbogens bezilglich der Annäherung durch Polygone, Acta Univ. Szeged. vol. 10 (1943) pp. 164-173. Starting from a different notion of deviation-the so-called deviation of area-among all convex arcs $A B$ lying in the interior of the triangle $A O B$ and enclosing a given area with the side $A B$, the conic arc touching $A O$ and $B O$ can be approximated worst. See $\mathrm{L}$. Fejes, Über eine Extremaleigenschaft der Kegelschnittbogen, Monatshefte für Mathematik und Physik vol. 50 (1943) pp. 317-326. 
perimeter the circle, more generally that among all continuously curved convex arcs of given length $l$ and total curvature $\omega$ the arc of a circle, can be approximated worst.

This is an immediate consequence of the explicit formula to be proved below, which expresses $a$ by the natural equation of $J$. We shall see that if the curvature $G$ of a rectifiable Jordan-curve $J$ of length $l$ is a continuous function of the length of arc $s$, then

$$
\frac{1}{a}=\frac{1}{8}\left(\int_{0}^{l}\left|G(s)^{v}\right|^{1 / 2} d s\right)^{2}
$$

where $a$ is the approximability of $J$ by inscribed polygons. ${ }^{4}$

Hence by Schwarz's inequality

$$
\frac{1}{a} \leqq \frac{1}{8} \int_{0}^{l} d s \cdot \int_{0}^{l}|G(s)| d s=\frac{1}{8} l \omega,
$$

where equality holds only in the case $G(s)=$ const., that is, when $J$ is an arc of a circle.

We shall prove our formula under the more general condition that $J$ is a skew curve. ${ }^{5}$ It is worthwhile to note that the torsion of the skew curve does not enter into our formula and hence the approximability is invariant under all transformations which do not alter the curvature as a function of the length of arc. ${ }^{6}$

First we estimate the deviation $\eta(p, j)$ of a chord $p$ from the corresponding arc $j$. For this purpose let us write the equations of $j$ in a coordinate system which coincides with the moving trihedron in the center of the arc $j$ of length $\sigma:^{7}$

$$
x_{1}=s+s^{2} \epsilon_{1}(s) \text {, }
$$

"We give two more analogous results without proof, and for the sake of simplicity only for the case of a closed convex curve $J: \lim n^{2}\left(t-t_{n}\right)=(1 / 12)\left(\int_{0}^{l} G^{1 / 3} d s\right)^{8}$, $\lim n^{2}\left(l-l_{n}\right)=(1 / 24)\left(\int_{0}^{l} G^{2 / 3} d s\right)^{3}$ where $t$ denotes the area of the oval surrounded by $J$, $t_{n}$ the area of the inscribed $n$-sided polygon having a maximal area, and $l_{n}$ the perimeter of the inscribed $n$-sided polygon having a maximal perimeter.

Let us observe that $\int_{0}^{l} G^{1 / 3} d s$ is the "affine length-of-arc" of $J$. (See, for example, W. Blaschke, Vorlesungen uber Differentialgeometrie, vol. 2, Berlin, 1923.)

We mention also without a proof that if we approximate a curve lying on a surface by polygons consisting of arcs of geodesic lines, we have to put into our formulas the geodetic curvature instead of $G$.

- This extension of my formula was first found by my pupil J. Horvath but his proof was not quite satisfactory.

- Such deformations of a curve can be illustrated on a simple model. See HilbertCohn-Vossen, Anschauliche Geometrie, Berlin 1932, footnote 2, p. 182.

${ }^{7}$ See, for example; A. R. Forsyth, Lectures on the differential geometry of curves and surfaces, Cambridge 1912, pp. 10-11. 


$$
\begin{aligned}
& x_{2}=2^{-1} G s^{2}+s^{2} \epsilon_{2}(s), \quad-\sigma / 2 \leqq s \leqq \sigma / 2, \\
& x_{3}=s^{2} \epsilon_{3}(s),
\end{aligned}
$$

where $G$ is the curvature at the considered point and $\epsilon_{1}(s), \epsilon_{2}(s), \epsilon_{8}(s)$ are continuous functions of $s$ which vanish for $s=0$.

Let us consider at the same time the arc $j^{\prime}$ whose equations are

$$
\begin{aligned}
& x_{1}=s, \\
& x_{2}=2^{-1} G s^{2}, \\
& x_{3}=0 .
\end{aligned}
$$

Obviously $\eta\left(j, j^{\prime}\right)$ can not be greater than the maximal distance of two points belonging to the same parameter:

$$
\begin{aligned}
\eta\left(j, j^{\prime}\right) & \leqq \max s^{2}\left(\epsilon_{1}^{2}+\epsilon_{2}^{2}+\epsilon_{3}^{2}\right)^{1 / 2} \\
& \left.\leqq(\sigma / 4) \max \left(\epsilon_{1}^{2}+{ }^{2} \epsilon_{2}+{ }^{2}\right)_{3}\right)^{1 / 2}=\left(\sigma^{2} / 4\right) \epsilon(\bar{\sigma}) ; \quad|\bar{\sigma}| \leqq \sigma / 2 .
\end{aligned}
$$

The deviation of the chord $p^{\prime}$ connecting both ends of $j^{\prime}$ from $p$ has the same upper bound: $\eta\left(p, p^{\prime}\right) \leqq\left(\sigma^{2} / 4\right) \epsilon(\bar{\sigma})$ and the deviation of $j^{\prime}$ from $p^{\prime}$ is obviously $\eta\left(p^{\prime}, j^{\prime}\right)=2^{-1} G \sigma^{2} / 4$.

Hence from the triangle inequalities

$$
\eta(j, p) \leqq \eta\left(j, j^{\prime}\right)+\eta\left(j^{\prime}, p^{\prime}\right)+\eta\left(p^{\prime}, p\right)
$$

and

$$
\eta\left(j^{\prime}, p^{\prime}\right) \leqq \eta\left(j^{\prime}, j\right)+\eta(j, p)+\eta\left(p, p^{\prime}\right)
$$

we get $8^{-1} G \sigma^{2}-2^{-1} \sigma^{2} \epsilon(\bar{\sigma}) \leqq \eta(p, j) \leqq 8^{-1} G \sigma^{2}+2^{-1} \sigma^{2} \epsilon(\bar{\sigma})$.

Let us consider now a sequence $P_{1}, P_{2}, \cdots$ of polygons inscribed into $J$ so that the greatest among the sides $p_{1}^{n}, p_{2}^{n}, \cdots, p_{n}^{n}$ of $P_{n}$ shall tend to zero with an increasing $n$. By the above inequality for any such sequence

$$
\lim _{n \rightarrow \infty} \sum_{i=1}^{n}\left(\eta\left(j_{i}^{n}, p_{i}^{n}\right)\right)^{1 / 2}=\frac{1}{8^{1 / 2}} \int_{0}^{l} G^{1 / 2} d s .
$$

Let us investigate the sequence of polygons for which

$$
\eta\left(p_{i}^{n}, j_{i}^{n}\right)=\eta\left(J, P_{n}\right) \quad i=1,2, \cdots, n ; n=1,2, \cdots .
$$

In this case

$$
\lim n\left(\eta\left(J, P_{n}\right)\right)^{1 / 2}=\frac{1}{8^{1 / 2}} \int_{0}^{l} G^{1 / 2} d s .
$$

As $\eta\left(J, P_{n}\right)$ is the maximal among the deviations $\eta\left(p_{i}^{n}, j_{i}^{n}\right)$, we get for 
every other sequence

$$
\lim n\left(\eta\left(J, P_{n}\right)\right)^{1 / 2} \geqq \frac{1}{8^{1 / 2}} \int_{0}^{l} G^{1 / 2} d s,
$$

assuming that the limit value on the left side exists. Therefore

$$
\lim \inf n\left(\eta\left(J, P_{n}\right)\right)^{1 / 2}=\frac{1}{8^{1 / 2}} \int_{0}^{l} G^{1 / 2} d s \quad \text { q.e.d. }
$$

We have seen that among all ovals in the plane with given perimeter the circle can be approximated worst. The question arises as to what can be said about the approximation by polygons consisting of arcs of circles.

Let us restrict ourselves to sketch only the main lines of the proof. For the detailed exposition the proof given in the preceding section can serve as an example.

We define the approximability $\alpha$ of a curve $J$ by inscribed circular arcpolygons by the limit value $1 / \alpha=\lim \inf n^{3} \eta\left(C_{n}, J\right)$ where $C_{n}$ denotes a polygon consisting of $n$ arcs of circles whose vertices lie on $J$.

Let $\bar{C}_{n}$ be the polygon whose deviation from $J$ is least for all polygons $C_{n}$. We may suppose that for the arcs of circles $c_{1}, c_{2}, \cdots, c_{n}$ of $\bar{C}_{n}$ and the corresponding arcs, $j_{1}, j_{2}, \cdots, j_{n}$ of $J$

$$
\eta\left(c_{i}, j_{i}\right)=\eta\left(\bar{C}_{n}, J\right), \quad i=1,2, \cdots, n,
$$

holds.

To estimate the deviation $\eta(c, j)$ we must also consider the members of third order in the canonical equations. Supposing $G^{\prime}(s)$ to be continuous, we have

$$
\begin{aligned}
& x_{1}=s-\sigma^{-1} G^{2} s^{3}+\cdots, \\
& x_{2}=2^{-1} G s^{2}+6^{-1} G^{\prime} s^{3}+\cdots .
\end{aligned}
$$

The equations of the circle of curvature at the same point are

$$
x_{1}=s-6^{-1} G^{2} s^{3}+\cdots, x_{2}=2^{-1} G s^{2}+\cdots
$$

If $G^{\prime} \neq 0$, the circle of curvature intersects the curve and the shortest distances of the circles of curvature from the points of $j$ with parameter values $s=\sigma / 2$ and $s=-\sigma / 2$ are $\sigma^{-1}\left|G^{\prime}\right|(\sigma / 2)^{3}+\cdots$. Let us turn the circle of curvature around the point of intersection so that the point with parameter value $s=\sigma / 2$ lies on $J$. In this case a point of the circle with parameter value $-\sigma / 2+\cdots$ lies on $J$ too, and two points of the arc $-\sigma / 2 \leqq s \leqq \sigma / 2$ of the circle of curvature with the parameter values $s= \pm\left(1 / 3^{1 / 2}\right)(\sigma / 2)+\cdots$ become the 
most remote ones from $J$, the maximal distance being $\left(3^{1 / 2} / 216\right)\left|G^{\prime}\right| \sigma^{8}$ $+\cdots$.

Consequently the arc $j$ can be approximated by a circular arc $c$ whose ends are coincident with the ends of $j$ so that

$$
\eta(c, j)=\frac{3^{1 / 2}}{216}\left|G^{\prime}\right| \sigma^{3}+\cdots
$$

On the other hand, it can be seen easily that this constant is exact.

Let us take the third root of these equalities involving the arcs $c_{i}$ of the polygon $\bar{C}_{n}$. By summing up and passing to the limit we get the theorem: Let $J$ be a plane curve with the natural equation $G=G(s), 0 \leqq s \leqq l$, where $G^{\prime}(s)$ is a continuous function. The reciprocal value of the approximability $\alpha$ of $J$ by inscribed circular arc polygons is

$$
\frac{1}{\alpha}=\frac{3^{1 / 2}}{216}\left(\int_{0}^{l}\left|G^{\prime}\right|^{1 / 3} d s\right)^{3} \text {. }
$$

Similarly it can be seen that this same $\alpha$ is the approximability of a skew curve by inscribed polygons consisting of arcs of screw lines. The approximability of a skew curve by inscribed circular arc polygons is

$$
\frac{1}{\alpha}=\frac{3^{1 / 2}}{216}\left(\int_{0}^{l}\left(\left|G^{\prime}\right|^{2}+G^{2} T^{2}\right)^{1 / 6} d s\right)^{3},
$$

where $T$ denotes the torsion.

Making use of Hölder's integral inequality ${ }^{8}$

$$
\left(\int_{a}^{b} \phi^{1 / 3} d x\right)^{3} \leqq(b-a)^{2} \int_{a}^{b} \phi d x ; \quad \phi(x) \geqq 0, a \leqq x \leqq b
$$

we get

$$
\frac{1}{\alpha} \leqq \frac{3^{1 / 2}}{216} l^{2} \int_{0}^{l}\left|G^{\prime}\right| d s
$$

which means that among the plane curves of monotone curvature with the length $l$ and maximal curvature $M$ the curve with the natural equation $G=(M / l)$ s can be approximated worst by polygons consisting of arcs of circles. This curve is the so-called clothoid ${ }^{9}$ (Cornu's spiral) which plays an important role in the theory of the interference of light.

Contrary to the approximability by common polygons, there is no

${ }^{8}$ See, for example, Hardy-Littlewood-Pólya, Inequalities, Cambridge, 1934.

๑ See, for example, G. Kowalewski, Natilrliche Geometrie, Berlin, 1931. 
oval of given perimeter which can be approximated worst by polygons of circular arcs; for, we can easily construct an oval with given perimeter out of clothoid arcs whose approximability $\alpha$ is arbitrarily small.

In the following we make no claim for rigour.

Let us consider an ovaloid $S$. We define the approximability $A$ of $S$ by inscribed polyhedra by the limit $1 / A=\lim \inf n \eta\left(Q_{n}, S\right)$, where $Q_{n}$ denotes an inscribed polyhedron having $n$ vertices. ${ }^{10}$

We assert that the approximability $A$ by inscribed polyhedra of an ovaloid $S$ with continuous Gauss measure of curvature $G$ can be expressed by the surface integral of $G^{1 / 2}$ :

$$
\frac{1}{A}=\frac{3^{1 / 2}}{9} \int_{S} G^{1 / 2} d f
$$

The same formula holds for the approximability by polyhedric surfaces of a part of an elliptically curved surface bordered by a continuously curved closed curve which does not cut itself. Thus the approximability is invariant under all deformations of the surface, that is, under the transformations which do not alter the line element at any point.

According to Schwarz's inequality

$$
\frac{1}{A} \leqq \frac{3^{1 / 2}}{9}\left(\int_{S} d f \cdot \int_{S} G d f\right)^{1 / 2}=\frac{3^{1 / 2}}{9}(F \Omega)^{1 / 2}
$$

where $\Omega=\int_{S} G d f$ is the total curvature of $S, F$ its area. Equality holds only for a surface which can be deformed into a part of a sphere of radius $(F / \Omega)^{1 / 2}$. Particularly: among all closed ovaloids with given area the sphere can be approximated worst. ${ }^{11}$

For the sake of simplicity we restrict ourselves to closed ovaloids. Let us consider a small triangle $\Delta$ whose vertices lie on $S$ and let $\Delta^{\prime}$ be the small part of $S$ lying near $\Delta$ whose normals intersect $\Delta$. Let us place $\Delta$ in the neighbourhood of a point $M$ of $S$, so that, fixing the

${ }^{10}$ We arrive at the same approximability $A$ by replacing the word "vertices" by "faces" or "inscribed" by "circumscribed" or both. The approximability by arbitrary polyhedra is $2 \mathrm{~A}$.

11 Starting from the notion of deviation of volume, the reciprocal value of the approximability is proportional to the square of the "affine surface." (L. Fejes, Über die isoperimetrische bzw. isepiphane Eigenschaft der Ellipse bzw. des Ellipsoids, Matematikai és Természettudományi Értesítö vol. 62 (1943) pp. 93-94; L. Fejes, Extremalis pontrendszerek a sikban, a gömbfelilleten es a terben, Acta Sci. Math. et Nat. vol. 23 (Kolozsvar 1944). Hence by the isepiphane property of the ellipsoid found by Blaschke (Vorlesungen iber Differentialgeometrie, vol. 2, pp. 198-201) the ellipsoid can be approximated worst among all ovaloids of given volume. 
deviation $\eta\left(\Delta, \Delta^{\prime}\right)$, the area of $\Delta$-which we denote by $\Delta$ too-shall be as great as possible. Let the plane drawn parallel to the tangential plane at $M$ at a distance $\eta$ meet $S$ in the Dupin's curve $D$. The position of $\Delta$ in question will be given by the maximal triangle inscribable in $D$.

In the first approximation $D$ can be considered as an ellipse with axes $\left(2 \eta R_{1}\right)^{1 / 2}$ and $\left(2 \eta R_{2}\right)^{1 / 2}$, where $R_{1}$ and $R_{2}$ are the two main radii of curvature at the point $M$. Thus the area of the maximal triangle inscribable in $D$ is

$$
\Delta_{\max }=\frac{3 \cdot 3^{1 / 2}}{4}\left(4 \eta^{2} R_{1} R_{2}\right)^{1 / 2}+\cdots,
$$

that is, for the triangle with area $\Delta$

$$
\eta\left(\Delta, \Delta^{\prime}\right)>\frac{2 \cdot 3^{1 / 2}}{9} G^{1 / 2} \Delta+\cdots .
$$

We can suppose that every face of the polyhedron $Q_{n}$ inscribed in $S$ is a triangle, since otherwise we could divide them into triangles. But a polyhedron consisting of triangles with $n$ vertices has $2 n-4$ faces. Thus summing up the above inequalities concerning the faces of $Q_{n}$ and increasing $n$ indefinitely we get

$$
2 \lim \inf n \eta\left(Q_{n}, S\right) \geqq \frac{2 \cdot 3^{1 / 2}}{9} \int_{S} G^{1 / 2} d f .
$$

On the other hand the bound on the right side can be attained by a suitably-chosen sequence of polyhedra; this completes the proof.

Let us note that the equality just proved does not hold for hyperbolically-curved surfaces. Consider, for example, the part $H$ of a hyperboloid of revolution of one sheet which lies between two planes parallel to its minimal circular section at the same distances on both sides. Let $A_{1} A_{2} \cdots A_{n}$ and $B_{1} B_{2} \cdots B_{n}$ be two regular polygons inscribable in the circles bordering $H$, so that $A_{i} B_{i}(i=1,2, \cdots, n)$ shall be generators of $H$. The deviation $\eta\left(H, Q_{2 n}\right)$ between $H$ and the polyhedric surface $Q_{2 n}$ having the faces $A_{1} A_{2} B_{1}, \cdots, A_{n} A_{1} B_{n}$, $B_{1} B_{2} A_{2}, \cdots, B_{n} B_{1} A_{1}$ is identical with the deviation between $A_{1} A_{2} \cdots A_{n}$ and the circle around it; hence its order of magnitude is $1 / n^{2}$ and not $1 / n$.

Finally we draw a conclusion from our estimation concerning $\eta\left(Q_{n}, S\right)$. Let $S$ be a sphere of radius $R$ and $Q_{n}$ a system of $n$ points on $S$. Write the greatest possible spherical calotte $s_{n}$ on $S$ which does not contain any point of $Q_{n}$. 
If we denote the radius of the circle at the base of $s_{n}$ by $r_{n}$ and the least convex covering of $Q_{n}$ by $Q_{n}$ too, then ${ }^{12}$

$$
\eta\left(Q_{n}, S\right)=R-\left(R^{2}-r_{n}^{2}\right)^{1 / 2} \text {. }
$$

For small values of $r_{n}$, this equals $r_{n}^{2} / 2 R+\cdots$ and thus by our estimation concerning $\eta\left(Q_{n}, S\right)$

$$
r_{n}^{2}>\frac{2 \cdot 3^{1 / 2}}{9} \frac{4 \pi R^{2}}{n}+\cdots
$$

or, supposing the system $Q_{n}$ lies on a part of $S$ with area $F$,

$$
r_{n}^{2}>\frac{2 \cdot 3^{1 / 2}}{9} \frac{F}{n}+\cdots
$$

It follows immediately that the upper bound of the radii of circles which does not contain any point of a plane system of points with density $d$ is

$$
r \geqq\left(\frac{2 \cdot 3^{1 / 2}}{9 d}\right)^{1 / 2} \text {. }
$$

This inequality is exact; equality holds for a lattice of equilateral triangles.

By the density of a system of points we understand here-provided that it exists-the limit value $d=\lim n(F) / F$, where $n(F)$ means the number of points in the region $F$ of area $F$ and the limit refers to the case when $F$ increases beyond all bounds by transformations of similitude.

Let us consider a system of circles of radius $\rho$ covering the plane. For the system of centres of circles obviously $r \leqq \rho$, hence the density of any system of congruent circles covering the plane is given by

$$
\pi \rho^{2} d \geqq \frac{2 \cdot 3^{1 / 2} \pi}{9} \quad(=1.209 \cdots) .
$$

This result is due to R. Kershner. ${ }^{13}$

BUdAPEST, Hungary

12 If the centre of the sphere $S$ lies outside $Q_{n}$ the square root in our equality must be taken with negative sign.

${ }^{13} \mathrm{R}$. Kershner, The number of circles covering a set, Amer. J. Math. vol. 61 (1939) pp. 665-671. For different generalizations of the above inequality see the second paper quoted in footnote 11 and further L. Fejes, Einige Bemerkungen ibber die dichteste Lagerung inkongruenter Kreise, Comment. Math. Helv. vol. 17 (1944-1945) pp. 256-261. 